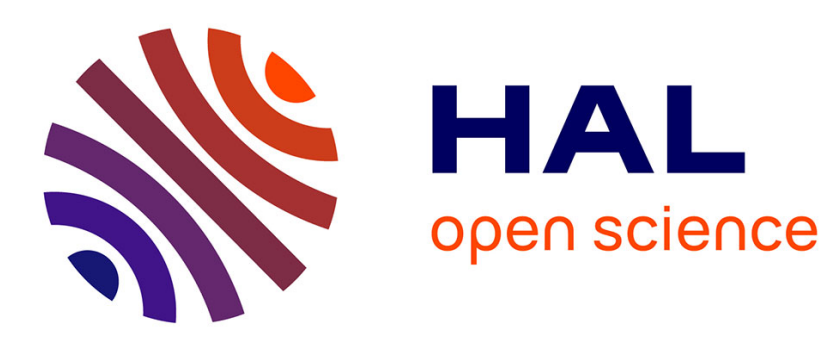

\title{
La séparation chez les couples corésidents de même sexe et de sexe différent
}

Benjamin Marteau

\section{To cite this version:}

Benjamin Marteau. La séparation chez les couples corésidents de même sexe et de sexe différent. Population (édition française), 2019, 74 (4), pp.521-549. 10.3917/popu.1904.0521 . hal-02483774

\section{HAL Id: hal-02483774 \\ https://hal.science/hal-02483774}

Submitted on 18 Feb 2020

HAL is a multi-disciplinary open access archive for the deposit and dissemination of scientific research documents, whether they are published or not. The documents may come from teaching and research institutions in France or abroad, or from public or private research centers.
L'archive ouverte pluridisciplinaire HAL, est destinée au dépôt et à la diffusion de documents scientifiques de niveau recherche, publiés ou non, émanant des établissements d'enseignement et de recherche français ou étrangers, des laboratoires publics ou privés. 


\title{
La séparation chez les couples corésidents de même sexe et de sexe différent
}

\begin{abstract}
Les études sur les couples de même sexe en Europe restent encore rares en raison du difficile repérage statistique de cette population. La connaissance sur la formation de ces couples s'est récemment enrichie avec l'accès, dans de nombreux pays, aux partenariats civils et au mariage. Mais la durée de vie de ces unions est encore assez méconnue. Se distingue-t-elle, ou non, de celle des couples de sexe différent? À partir des données du programme international d'enquêtes Générations et genre (GGS), l'auteur compare dans six pays européens le risque de séparation des couples gays et des couples lesbiens corésidents relativement aux couples de sexe différent, en tenant compte des particularités de chacun.
\end{abstract}

Les études scientifiques consacrées à la stabilité et la durée des couples ont commencé à se développer dans les années 1950. Des changements dans le système de valeurs des sociétés occidentales s'y opèrent alors, se traduisant par une sécularisation progressive du couple, une augmentation des divorces et une baisse de la fécondité. Ces évolutions sociétales ont mené les chercheurs à s'interroger sur les facteurs qui favorisaient ou non la longévité du mariage. Par la suite, la multiplication des remariages ou des remises en union consécutive à l'augmentation des divorces, et les nouveaux modes de vie en couple (hors mariage, non-cohabitation, familles recomposées) ont conduit au développement de nouveaux cadres théoriques pour expliquer ces changements, par exemple celui de la « seconde transition démographique » (Van De Kaa, 1987; Lesthaeghe, 2010).

La majorité de ces travaux se concentrent sur des couples composés d'un homme et d'une femme, négligeant les couples de même sexe. Certaines études sur la stabilité des unions n'en mentionnent même pas l'existence

\footnotetext{
* Institut national d'études démographiques (Ined); Centre de recherche en démographie, Université Catholique de Louvain (UCL).

Correspondance : Benjamin Marteau, Institut national d'études démographiques (Ined), F-75020 Paris, courriel: benjamin.marteau@ined.fr
} 
(Becker, 1981; Oppenheimer, 1988), tandis que d'autres précisent que leurs observations se limitent aux couples hétérosexuels (Brines et Joyner, 1999). Les premières analyses sur les unions de partenaires de même sexe remontent aux années 1980 et se concentrent surtout aux États-Unis, à partir de données d'enquêtes à petite échelle ou d'études qualitatives (Blumstein et Schwartz, 1983; Kurdek, 1998).

Ce manque d'attention pour les couples gays et lesbiens dans les travaux sur la stabilité des unions provient notamment du fait que l'union civile ou le mariage homosexuel n'ont été légalisés qu'à partir des années 1990 pour les pays précurseurs comme le Danemark ou la Norvège, et quelques années plus tard dans d'autres pays occidentaux. Cela a eu pour conséquence de limiter les recherches sur ce sujet, vu le peu de visibilité de ces couples dans les données statistiques et l'absence de cadre légal dont ils bénéficiaient. En effet, les couples homosexuels ne représentent qu'une minorité de la population, et les sources de données à disposition ne permettent pas toujours de clairement les identifier ou d'en estimer le nombre.

Désormais, l'accès au mariage, à la parentalité et à une reconnaissance sociale des couples de même sexe est possible. Toutefois, les facteurs de stabilité ou d'instabilité de ces couples restent peu explorés. Or, les couples de même sexe ont des caractéristiques sociodémographiques qui se distinguent des couples de sexe différent, pouvant se répercuter sur leurs dynamiques conjugales. Alors que certaines caractéristiques ont un effet positif sur la longévité des couples de sexe différent, celles-ci pourraient se refléter différemment chez les couples de même sexe. Dès lors, deux principales questions de recherche se posent dans cette étude : les couples de même sexe présentent-ils un risque plus important de se séparer que les couples de sexe différent? Quels sont les facteurs associés à ces risques de rupture?

Les données de l'enquête européenne Generations and Gender Survey (GGS) sont exploitées pour plusieurs pays (Allemagne, Belgique, France, Norvège, Pays-Bas, Suède) et les parcours conjugaux des individus interrogés sont reconstitués afin de comparer la longévité des couples de même sexe $(\mathrm{N}=385)$ et des couples de sexe différent $(\mathrm{N}=36090)$ formés depuis les années 1980.

Après avoir évoqué les principales théories et facteurs influençant la stabilité du couple, cet article présentera une synthèse des quelques études quantitatives ayant déjà traité des couples de même sexe. Nous expliciterons ensuite comment le couple est défini dans l'enquête GGS, ainsi que l'histoire conjugale des individus en couple corésident. Un modèle de Cox permettra d'analyser les risques de séparation en tenant compte des caractéristiques des individus et des couples. 


\section{Deux types de couples aux caractéristiques spécifiques}

\section{Une littérature marquée par le contexte légal}

Jusqu'à la fin des années 1990, la plupart des recherches sociodémographiques sur la stabilité du couple ne portent que sur les couples hétérosexuels ${ }^{(1)}$. Puis, certains chercheurs ont élargi ces recherches aux couples homosexuels (Andersson et al., 2006; Lau, 2012; Wiik et al., 2014; Manning et al., 2016), en prenant en compte le contexte social et légal qui a évolué pour les deux types de couples. Ces recherches comparent fréquemment la longévité des couples de même sexe à celle des couples de sexe différent. Les tendances générales qui en ressortent sont des risques de rupture plus élevés pour les partenaires de même sexe, bien que des nuances soient nécessaires, compte tenu des effets de structure propres à ces unions.

Parmi les couples de sexe différent qui vivent ensemble, les couples cohabitants mariés se distinguent des couples cohabitants non mariés, qui diffèrent dans leurs attentes envers leurs partenaires. Les personnes en couple non marié ont tendance à être plus individualistes et à rechercher une relation égalitaire avec leur partenaire, notamment dans le partage des tâches ménagères ou des revenus, remettant en cause les modèles de genre « traditionnels » (Brines et Joyner, 1999; Weisshaar, 2014). La spécialisation en matière de division du travail réalisée au sein du mariage est donc plus conséquente et augmente la dépendance entre les conjoints mariés. Les coûts d'un divorce peuvent être un frein à la séparation, surtout pour les femmes qui sont soumises à une détérioration plus importante de leur niveau de vie que les hommes après le divorce (Andress et al., 2006). L'effet causal de la contractualisation de l'union sur le risque de séparation n'a toutefois pas été démontré directement (Blumstein et Schwartz, 1983; Brines et Joyner, 1999). Bien que la proportion de couples non mariés augmente dans de nombreux pays occidentaux, ce modèle reste associé à une probabilité de rupture supérieure par rapport aux couples mariés ou en union civile (Brines et Joyner, 1999; Blackwell et Lichter, 2004; Lyngstad et Jalovaara, 2010).

Étant donné que l'accès au mariage ne leur a pas toujours été possible, le contexte légal et normatif pour les couples de même sexe n'est pas le même que celui des couples de sexe différent. Les unions homosexuelles ne sont reconnues dans certains pays que depuis deux décennies, et sous diverses formes (union civile, mariage, parfois les deux). La plupart des couples de même sexe, s'ils vivent ensemble, n'ont pas toujours été en mesure de contractualiser leur union (Banens, 2010; Rosenfeld, 2014). Cette non-contractualisation de l'union, dans le cas où elle a un effet similaire à celui sur les couples de sexe différent, pourrait alors déterminer en grande partie le risque plus élevé de séparation des couples de même sexe.

(1) Le terme « couple hétérosexuel » n'est pas utilisé dans ce travail en référence à l'orientation sexuelle, il est considéré comme un synonyme du terme " couple de sexe différent ». Il en est de même pour le terme « couple homosexuel » et « couple de même sexe ». 
Enfin, pour ces couples de même sexe, la possibilité de donner une dimension légale à l'union ne signifie pas qu'ils le feront nécessairement. L'usage, selon les pays, est varié : la Norvège et la Suède sont caractérisées par de faibles taux de contractualisation, alors que ces taux sont élevés au Royaume-Uni (Banens, 2010). Le rapport à l'engagement dans la conjugalité, au-delà de la contractualisation de l'union, peut toutefois s'établir d'une autre manière. Le fait de vivre ensemble sous le même toit par exemple, phénomène moins fréquent pour les unions homosexuelles qu'hétérosexuelles, témoigne de normes conjugales différentes entre les deux types de couples (Rault, 2018; RégnierLoilier, 2018b). Charles Lau, dans son étude sur la stabilité des couples au Royaume-Uni, a montré que les partenaires de même sexe en cohabitation non mariés présentent des risques de rupture légèrement plus élevés que les couples non mariés de sexe différent. Les couples mariés hétérosexuels sont ceux qui ont le plus de chances de durer (Lau, 2012). Mais le contexte national peut aussi avoir une influence, puisque certaines études aux États-Unis fournissent d'autres informations, notamment le fait qu'un risque de rupture peut être identique entre les couples non mariés de même sexe et ceux de sexe différent (Manning et al., 2016).

\section{La présence d'enfant(s)}

La présence d'un enfant au sein du couple est un autre élément mis en évidence dans la plupart des recherches sur la longévité des couples, comme un frein à la séparation (Levinger, 1965). Un sentiment moral ou d'obligation envers l'enfant peut faire obstacle au risque de séparation. Mais l'augmentation des séparations de couples avec enfants et des familles recomposées ensuite laisse penser que ce sentiment moral s'estompe avec le temps. Les couples sans enfant ont également des caractéristiques particulières pouvant être associées à des risques de séparation plus élevés : ils ont, en général, des valeurs moins conservatrices par rapport à la famille, ne sont souvent pas mariés ou ont un faible attachement religieux (Lyngstad et Jalovaara, 2010).

Au sein des couples de même sexe, la présence d'enfants est rare, surtout chez les hommes qui font face à des inégalités d'ordre légal et biologique pour devenir parents, ainsi qu'à des procédures d'adoption plus restrictives que les couples de sexe différent. Les couples de femmes ont en moyenne plus d'enfants, qu'ils soient issus d'une précédente relation ou d'une insémination artificielle, et sont en conséquence davantage soumis à ce sentiment moral de préservation du couple pour les enfants (Gross et al., 2014). En présence d'enfants, les couples d'hommes seraient plus fragiles que les couples de femmes (Wiik et al., 2014). Quant aux enfants issus d'une précédente relation, ils seraient généralement associés à un risque accru de se séparer, tandis que ceux du couple actuel seraient vus comme augmentant la longévité du couple (Lyngstad et Jalovaara, 2010; Wiik et al., 2014). 


\section{L'hétérogamie chez les couples de même sexe}

Il est aussi important de noter que les possibilités de rencontre pour les personnes homosexuelles sont réduites, par comparaison aux personnes hétérosexuelles qui trouvent souvent un conjoint ou une conjointe sur leur lieu de travail ou d'études (Blackwell et Lichter, 2004; Bozon et Rault, 2012). Comme certains travaux l'ont déjà montré, les écarts en termes d'âge ou de profil socioéconomique entre deux partenaires de même sexe sont plus élevés que chez des conjoints de sexe différent. Cela peut participer aux écarts en matière de risques de séparation, l'hétérogamie étant d'ordinaire associée à une moindre stabilité (Andersson et al., 2006; Kalmijn et al., 2007; Verbakel et Kalmijn, 2014).

\section{Hypothèses de recherche}

L'ensemble des études déjà réalisées sur la durée des unions semble donc indiquer que, de manière générale, les unions de même sexe présentent plus de risques de séparation que les unions de sexe différent (HI). L'enquête GGS permettra d'élargir le champ d'études à plusieurs pays d'Europe et de tester cette hypothèse.

Le mariage (ou le partenariat enregistré) est à la fois plus courant et plus ancien chez les couples de sexe différent que chez ceux de même sexe. De ce fait, il peut ne pas être vécu de la même façon. Il correspond à des temporalités spécifiques en raison de la possibilité de formaliser ou pas l'union en mariage pour les couples de même sexe. Emménager avec un partenaire, sans pour autant formaliser l'union, peut constituer une étape importante dans l'engagement conjugal chez les couples de même sexe, sachant qu'ils vivent plus régulièrement en couple non cohabitant que les couples de sexe différent. La formalisation de l'union pourrait dès lors revêtir un caractère moins symbolique que chez les couples de sexe différent, et être associé à des risques de séparation différenciés. La deuxième hypothèse est que l'effet stabilisateur du mariage (ou du partenariat) est moins fort chez les couples de même sexe que chez les couples de sexe différent (H2).

La parentalité est un autre facteur susceptible d'influer sur le risque de séparation, dont l'effet pour les couples de même sexe n'a pas encore été clairement démontré. Les projets de parentalité sont difficiles à réaliser pour ces couples, et les enfants sont souvent issus d'une précédente relation. Le frein à la séparation que constituent les enfants est ainsi moins présent dans ces caslà. Les couples de même sexe sans enfant et les couples de sexe différent sans enfant pourraient dès lors se ressembler, ce qui permet de dégager une dernière hypothèse, selon laquelle les couples de même sexe et de sexe différent sans enfant présentent des risques similaires de séparation (H3). 


\section{Données et contexte}

\section{Données}

L'enquête Generations and Gender Survey (GGS) est une enquête longitudinale (trois vagues d'enquêtes conduites à trois ans d'intervalle) ayant pour but de récolter des données dans plusieurs pays développés afin de comprendre les causes et conséquences des changements démographiques.

La population-cible de GGS est la population résidente dans des ménages « ordinaires » (non collectifs), âgée de 18 à 79 ans lors de la première vague d'enquête $^{(2)}$. Six pays sont sélectionnés pour notre étude : l'Allemagne, la Belgique, la France, la Norvège, les Pays-Bas et la Suède. L'enquête GGS renseigne sur le parcours conjugal des individus, avec des questions sur les unions cohabitantes actuelles ou précédentes du répondant. Le choix s'est porté sur ces pays en raison de la présence de la variable « sexe du partenaire » dans leur questionnaire, qui nous permet d'étudier les couples de même sexe. De plus, la relative tolérance envers l'homosexualité dans ces pays limite la proportion d'individus qui n'oseraient pas déclarer vivre ou avoir vécu avec un partenaire du même sexe, bien que le stigmate social puisse encore être en partie présent chez certaines personnes. La proximité géographique de ces pays ne signifie pas une homogénéité culturelle, c'est pourquoi nous contrôlons l'effet du pays dans nos modèles statistiques. La combinaison de plusieurs enquêtes nationales est cependant indispensable dans notre cas afin d'obtenir un échantillon suffisant de couples corésidents de même sexe, très minoritaires au sein de la société et dans les enquêtes nationales représentatives de la population. Seule la première vague des enquêtes est exploitée dans cette recherche.

\section{Contexte}

En Europe, un statut légal pour les couples de même sexe s'est progressivement mis en place dans plusieurs pays du continent, laissant la possibilité à ceux qui le désiraient de contractualiser leur relation (tableau 1). L'usage et la symbolique de ce statut diffèrent selon les pays.

Dans l'enquête GGS, seul le partenariat enregistré en France (pacs) est repérable dans les données comme un statut d'union spécifique, distinct du mariage et de l'union libre. Comme aux Pays-Bas, le partenariat enregistré en France est ouvert aux couples de même sexe et de sexe différent. En Belgique, la cohabitation légale est possible pour deux personnes cohabitantes, quels que soient leur sexe et leurs liens familiaux. Elle est une manière pour les couples homosexuels de formaliser leur union. Dans ces trois pays, même après l'ouverture des droits du

(2) Chaque pays choisit sa propre méthode d'échantillonnage, ce qui amène une variabilité lorsque les données de plusieurs pays sont assemblées. Dans cet article, les pondérations des enquêtes nationales sont utilisées. Pour plus de détails, voir l'article sur la collecte de données de Fokkema et al. (2016). 
Tableau 1. Statuts légaux pour les couples de même sexe dans les pays d'enquête

\begin{tabular}{|c|c|c|c|c|}
\hline Pays & Nom & $\begin{array}{l}\text { Période de mise en } \\
\text { application }\end{array}$ & $\begin{array}{c}\text { Année d'enquête GGS } \\
\left(1^{\text {re }} \text { vague }\right)\end{array}$ & $\begin{array}{c}\text { Information } \\
\text { disponible dans GGS }\end{array}$ \\
\hline Belgique & $\begin{array}{l}\text { Cohabitation légale } \\
\text { Mariage }\end{array}$ & $\begin{array}{l}2000- \\
2003-\end{array}$ & $2008-2010$ & $\begin{array}{l}\text { Non } \\
\text { Oui }\end{array}$ \\
\hline France & $\begin{array}{l}\text { Pacs } \\
\text { Mariage }\end{array}$ & $\begin{array}{l}1999- \\
2013-\end{array}$ & 2005 & $\begin{array}{l}\text { Oui } \\
\text { Non }\end{array}$ \\
\hline Pays-Bas & $\begin{array}{l}\text { Partenariat enregistré } \\
\text { Mariage }\end{array}$ & $\begin{array}{l}1998- \\
2001-\end{array}$ & $2002-2004$ & $\begin{array}{l}\text { Non } \\
\text { Oui }\end{array}$ \\
\hline Allemagne & $\begin{array}{l}\text { Partenariat enregistré } \\
\text { Mariage }\end{array}$ & $\begin{array}{c}2001-2017 \\
2017-\end{array}$ & 2005 & $\begin{array}{l}\text { Non } \\
\text { Non }\end{array}$ \\
\hline Norvège & $\begin{array}{l}\text { Partenariat enregistré } \\
\text { Mariage }\end{array}$ & $\begin{array}{c}1993-2009 \\
2009-\end{array}$ & $2007-2008$ & $\begin{array}{l}\text { Oui } \\
\text { Non }\end{array}$ \\
\hline Suède & $\begin{array}{l}\text { Partenariat enregistré } \\
\text { Mariage }\end{array}$ & $\begin{array}{c}1995-2009 \\
2009-\end{array}$ & $2012-2013$ & $\begin{array}{l}\text { Oui } \\
\text { Oui }\end{array}$ \\
\hline
\end{tabular}

mariage aux couples de même sexe, les unions civiles restent une possibilité pour tous les couples de contractualiser leur union.

La signification du «partenariat enregistré » est différente en Suède et en Norvège. Avant 2009, il était réservé uniquement aux couples de même sexe. Il accordait alors des droits similaires à ceux des mariages de couples de sexe différent, et était présenté comme équivalent (Andersson et al., 2006). Dans les données utilisées ici, il sera donc assimilé à un mariage. À partir de 2009, le partenariat enregistré dans ces deux pays disparaît au profit du mariage pour les nouveaux couples de même sexe, afin d'obtenir la même dénomination et les mêmes dispositions légales que pour les couples de sexe différent (Banens, 2010; Wiik et al., 2014).

Enfin, en Allemagne, le partenariat enregistré n'est ouvert que pour les couples de même sexe, sans accorder autant de droits que dans le modèle nordique (Festy, 2006).

$\mathrm{Au}$ moment de la première vague d'enquête GGS, on peut considérer que la Belgique, la Norvège, les Pays-Bas et la Suède avaient ouvert un accès au mariage (ou un équivalent proche) pour les couples de même sexe. En France, seul le pacs était disponible pour formaliser l'union. En Allemagne, le partenariat enregistré était légal au moment de l'enquête mais l'information n'est pas présente dans GGS.

\section{Méthodologie}

\section{Définition du couple et de l'histoire conjugale}

L'enquête GGS interroge un seul membre des ménages de l'échantillon, mais récolte quelques informations sur les autres membres. La présence 


\section{Encadré 1. L'identification des couples de même sexe dans GGS}

Le croisement des variables correspondant au sexe du répondant et du partenaire ne suffit pas à repérer les couples de même sexe dans l'enquête GGS. Des erreurs d'encodage existent, et même si le taux d'erreur est peu élevé, la faible proportion de couples homosexuels dans la population peut être fortement affectée par ces biais de saisie des données (Festy, 2007).

Des vérifications peuvent être apportées pour identifier et réduire les biais liés aux " faux » couples de même sexe. Tout d'abord, la date de mariage pour les couples mariés qui doit avoir lieu après la légalisation du mariage homosexuel dans les pays concernés. Pour les couples mariés, apparemment de même sexe, si la date de mariage est antérieure à cette légalisation, le recodage en couples de sexe différent s'effectue. Un deuxième niveau de vérification porte sur le fait d'avoir un enfant biologique avec son partenaire actuel au sein du ménage, ce qui est peu (non) probable dans le cas de deux personnes du même sexe. Enfin, un dernier élément pris en compte est la réponse à une question d'opinion, à savoir : " les couples homosexuels devraient avoir les mêmes droits que les couples hétérosexuels? ". Pour les individus répondant ne pas (du tout) être d'accord avec cette affirmation, ceux-ci sont également recodés en couple de sexe différent. Cette vérification permet d'éliminer 145 " fausses " unions de même sexe sur 530, pour les unions formées après 1980 et pour les individus âgés au moment de l'enquête de 60 ans et moins (tableau 2).

Tableau 2. Nombre de couples de même sexe et pourcentage d'erreurs d'encodage du sexe, par pays

\begin{tabular}{|l|c|c|c|c|c|c|}
\hline \multicolumn{1}{|c|}{ Pays } & $\begin{array}{c}\text { N couples de } \\
\text { même sexe }\end{array}$ & \% Mariage & $\begin{array}{c}\text { \% Enfants } \\
\text { biologiques }\end{array}$ & \% Opinion & $\begin{array}{c}\text { N couples de } \\
\text { même sexe après } \\
\text { correction }\end{array}$ & $\begin{array}{c}\text { \% d'erreur } \\
\text { total }\end{array}$ \\
\hline Belgique & 81 & 16,1 & 2,5 & 2,5 & 64 & 20,1 \\
Allemagne & 73 & 46,6 & 4,1 & 1,4 & 35 & 52,1 \\
Pays-Bas & 123 & 11,4 & 4,9 & 0,0 & 103 & 16,3 \\
Norvège & 87 & 0,0 & 3,5 & 6,9 & 78 & 10,4 \\
Suède & 60 & 1,7 & 5,0 & 0,0 & 56 & 6,7 \\
France & 106 & 23,6 & 9,4 & 8,5 & 62 & 41,5 \\
France & 106 & $/$ & $/$ & $/$ & 49 & 53,8 \\
(prénom) & 530 & 16,4 & 5,1 & 3,4 & 385 & 24,9 \\
\hline Total & & & & & & \\
\hline
\end{tabular}

Note : Les types d'erreurs sont pris l'un à la suite de l'autre, un seul couple peut comporter une erreur à la fois sur la date de mariage et par rapport aux enfants biologiques.

Lecture : 16,1 \% des couples de même sexe en Belgique sont repérés comme erronés après vérification de la date de mariage.

Champ : Couples corésidents formés dès 1980, par des individus âgés de 60 ans et moins.

Sources: GGS Vague 1, GGS Vague 1 France (base de données des prénoms des répondants et de leur(s) partenaire(s)).

habituelle d'un partenaire dans le logement permet de considérer l'union comme corésidente. En dehors de sa relation au moment de l'enquête, le répondant décrit toutes ses précédentes relations corésidentes. Aucune référence n'est faite aux relations antérieures qui n'ont pas impliqué le partage d'un domicile commun. Il n'est également pas possible de connaître la date à laquelle le couple s'est rencontré, avant de s'installer dans un même domicile.

Les unions libres - des couples sans contrat de vie commune ou non mariés - sont incluses dans l'histoire conjugale. Les couples de droit comme 


\section{Encadré 1 (suite). L'identification des couples de même sexe dans GGS}

La Norvège et la Suède, partant de leurs registres de population pour collecter les informations sociodémographiques de base sur les individus échantillonnés, comprennent moins d'erreurs concernant le sexe des individus ou de leurs partenaires. La France et l'Allemagne présentent des taux d'erreurs plus importants. Ces deux pays, ainsi que la Belgique, se caractérisent par une méthode de collecte de données reposant uniquement sur la collecte en face-à-face, par un agent enquêteur, tandis que les Pays-Bas combinent cette collecte à des questionnaires auto-administrés (Fokkema et al., 2016). Ceci peut expliquer les erreurs d'encodage plus élevées que dans des pays ayant recours à des bases de données administratives, plus fiables sur les données du sexe des individus.

En France, l'accès à une base de données sur les prénoms des répondants et de leurs partenaires a permis de réaliser une correction alternative plus précise concernant les faux couples de même sexe. Cette correction est privilégiée dans nos analyses ultérieures. Au vu du pourcentage d'erreurs obtenu en France à partir de la base de données des prénoms par rapport aux autres pays, il est probable que la correction effectuée ne couvre pas l'ensemble des faux couples de même sexe, notamment en Belgique et aux Pays-Bas où le pourcentage d'erreur semble faible par rapport à la France et à l'Allemagne disposant à peu près du même protocole de collecte de données. Dans le questionnaire des Pays-Bas notamment, la question d'opinion n'est pas présente, enlevant les possibilités d'exclure des unions à partir de cet item.

de fait sont donc renseignés dans cette partie du questionnaire, élément particulièrement important dans le cas des couples de même sexe qui ne disposent que depuis peu de l'opportunité de donner un statut légal à leur union.

\section{Description des variables}

La variable dépendante de nos analyses statistiques est la rupture de l'union. Dans les modèles longitudinaux mesurant les risques de rupture selon la durée d'union, chaque couple est suivi dès le début de sa relation corésidente, si elle a lieu après $1980^{(3)}$, jusqu'à la date de sa séparation éventuelle. Pour les couples mariés, c'est la date de fin de vie commune qui est utilisée comme indicateur de séparation, pas la date de divorce légalement reconnu. Le champ d'étude est limité aux individus âgés de 60 ans et moins afin de diminuer le risque de sous-déclaration d'anciennes unions.

La variable indépendante principale est la composition sexuée du couple (ou type de couple), à savoir si l'union est formée de deux individus du même sexe ou de sexe différent. Des erreurs d'encodage sont à déplorer concernant le sexe des individus ou de leur partenaire, ce qui peut surestimer le nombre de couples homosexuels enregistrés dans la base de données (Festy, 2007). L'encadré explique la vérification (et la correction) proposée, qui ne peut néanmoins assurer l'élimination de toutes les erreurs (Régnier-Loilier, 2018a).

(3) La restriction de la période aux unions formées après 1980 permet de mieux comparer les couples de même sexe et de sexe différent, puisque peu de couples de même sexe se sont formés avant les années 1980 . 
Des variables indépendantes et de contrôle sont également intégrées aux modèles :

- La contractualisation de l'union, qui permet de vérifier la deuxième hypothèse sur le risque différencié du mariage sur les deux types de couples. Comme certains couples vivent ensemble avant de se marier, cette donnée est considérée comme variant dans le temps dans tous les modèles. Les partenariats enregistrés, lorsqu'ils sont repérables dans l'enquête (en France) ou qu'ils sont un équivalent au mariage pour les couples de même sexe (en Suède et Norvège), sont considérés comme une forme de contractualisation de l'union.

- La présence et le nombre d'enfants (dans le ménage ou non) servent à vérifier la troisième hypothèse sur le risque de séparation accru en l'absence d'enfant pour les deux types de couples. Dans le modèle, nous distinguons deux mesures de la parentalité, à savoir la naissance d'un enfant au cours de l'union ou l'existence d'un enfant en début d'union ${ }^{(4)}$. La première mesure est dépendante du temps tandis que la seconde est fixe. Les enfants communs du couple, qu'ils soient résidents ou non dans le ménage au moment de l'enquête, incluent les enfants biologiques (et ceux adoptés dans le cas de la Suède). Pour les anciennes unions déclarées dans l'histoire conjugale, l'hypothèse est que les enfants nés après que les partenaires aient commencé à vivre ensemble sont des enfants provenant de cette union. Les enfants issus d'une précédente union regroupent les enfants nés d'une ancienne relation du partenaire ou ceux du répondant.

- La disponibilité d'un cadre légal pour les couples de même sexe. Selon la période et le pays, celui-ci n'est pas toujours accessible, et la modalité de contractualisation de l'union, quand elle est disponible dans l'enquête, n'introduit alors que de l'hétérogénéité pour les couples de sexe différent. Cette variable dépendante du temps permet ainsi de rendre compte de la période et de la possibilité qu'ont les partenaires de même sexe de se marier (se pacser) ou non.

- La différence d'âge entre les partenaires ${ }^{(5)}$.

- Lâge d'entrée en union du répondant.

- Le processus de transmission intergénérationnelle de la séparation, mesuré par l'éventuelle séparation des parents du répondant avant l'âge de 18 ans.

(4) Pour les identifier, nous comparons l'année de naissance des enfants et celle de la mise en union, ainsi que les liens avec les membres du ménage ou d'éventuels enfants ne résidant pas dans celuici. Pour les anciennes relations, une question est posée au répondant, lui demandant si son ancien partenaire avait des enfants au moment de commencer leur union.

(5) Lâge des anciens partenaires corésidents n'est pas demandé en Norvège et aux Pays-Bas, il est imputé aléatoirement de telle sorte qu'il approche la distribution obtenue dans les autres pays. 
- Le rang de l'union du répondant distingue les premières unions cohabitantes et les unions de rang supérieur.

- Lâge des répondants au moment de l'enquête.

- Le niveau d'instruction du répondant.

- Le pays de résidence.

\section{Modèles d'analyse statistique et biographique}

\section{Vérification de la cohérence du parcours conjugal}

Des erreurs de date et des valeurs manquantes dans le parcours conjugal des individus, associées à la plupart des enquêtes de ce type (en de faibles proportions), introduisent des biais dans les modèles, que nous devons dès lors traiter.

Ce type d'erreur (valeurs manquantes, date de début d'union après la date de séparation...) concerne 4,2\% des individus de moins de 61 ans de l'échantillon, soit 37443 personnes. Les 1503 individus présentant des incohérences de datation dans leur parcours conjugal sont éliminés. Léchantillon exploité est donc constitué de 35940 individus pour un total de 46861 unions. En ne tenant compte que des unions formées après 1980, l'échantillon est réduit à 28347 individus pour 36475 unions.

Lunité de temps utilisée pour les analyses est celle du mois. La date de formation ou de séparation de l'union est renseignée au mois près, lorsque l'individu est capable de s'en souvenir. Certains mois ont été imputés, notamment lorsque la saison était indiquée si la personne ne précisait pas le mois exact. Les mois manquants sans indication d'une période de temps approximative ont été imputés aléatoirement selon une répartition uniforme, en veillant au maintien de la cohérence du parcours conjugal. Lorsque l'union s'est formée et rompue dans la même année, l'imputation fait en sorte que l'union commence plus souvent en début d'année et se termine en fin d'année.

\section{Analyse descriptive des biographies}

Après les corrections d'erreurs d'encodage du sexe et de datation, un modèle de survie de l'union a été construit. La fonction de survie de base équivaut à un estimateur non paramétrique de Kaplan-Meier. Il est possible de différencier les fonctions de survie pour les couples de même sexe ou les couples de sexe différent, et de connaître le nombre de mois avant lequel un pourcentage défini de couples s'est séparé. Ensuite, un quotient de séparation est calculé comme le nombre de séparations d'unions corésidentes dans une période donnée divisé par l'ensemble des unions à risque en début de période. Ces quotients sont calculés par période de 6 mois d'union.

Lanalyse descriptive des biographies indique donc des risques de séparation selon la durée de l'union et le type de couple. Comme l'intérêt porte ici sur les unions corésidentes, le début de ces unions est marqué par l'emménagement 
des deux conjoints dans un logement commun, quelle que soit la durée passée au préalable en couple non cohabitant.

\section{Modèle de Cox à risque proportionnel}

Des modèles d'analyse longitudinale incluant des facteurs explicatifs sont par la suite développés. Un modèle de Cox portant sur chaque union des individus interrogés dans l'enquête est construit. La fonction de risque de connaître une séparation est conditionnée par des variables explicatives fixes et dépendantes du temps. Les termes d'erreurs sont groupés par individu, puisqu'un individu peut connaître plusieurs épisodes de couple.

Les deux premiers modèles créés n'incluent que la variable indépendante d'intérêt à savoir le type de couple (de même sexe, de sexe différent), tandis que les suivants intègrent tour à tour les variables indépendantes et les variables de contrôle. Un terme d'interaction entre contractualisation de l'union et type de couple permet d'évaluer l'effet spécifique du mariage selon la composition sexuée de l'union. Le même procédé est utilisé pour évaluer l'influence de la présence d'enfants en commun ou issus d'une précédente relation.

\section{Résultats}

\section{Caractéristiques des unions et des individus interrogés}

Le tableau 3 reprend les caractéristiques de l'échantillon et des couples corésidents de même sexe $(\mathrm{N}=385)$ ou de sexe différent $(\mathrm{N}=36090)$. La majeure partie des répondants en couple sont toujours dans leur première union au moment de l'enquête.

Dans l'ensemble des unions formées à partir de 1980, 1,05 \% sont de même sexe, quelle que soit la période de formation des couples. L'année de début d'union joue un rôle quant aux chances de cohabiter pour deux partenaires de même sexe, plus fréquentes pour les périodes récentes. Il est compliqué de déterminer si ces chiffres correspondent à ceux d'autres enquêtes, car très peu d'études portant sur les individus en couples de même sexe sont disponibles en Europe. La plupart des chiffres officiels pour comparer ces résultats portent uniquement sur les unions enregistrées légalement. En Norvège par exemple, 0,7\% des partenariats enregistrés entre 1993 et 2001 ont été contractés par des partenaires de même sexe. C'est le cas de 0,5\% des partenariats en Suède entre 1995 et 2002 (Andersson et al., 2006). Dans leur étude sur l'homogamie des couples de même sexe aux Pays-Bas entre 2001 et 2007, Verbakel et Kalmijn (2014) établissent cette proportion à 0,9\% (couples mariés et non mariés inclus). En France, à partir de l'enquête Famille et logements adossée au recensement de 2011, on compte 0,6 \% de couples (corésidents ou non) de même sexe (Buisson et Lapinte, 2013). Dans notre échantillon, il y a à la fois des couples 
Tableau 3. Caractéristiques des unions (\%) composées de deux partenaires du même sexe ou de sexe différent

\begin{tabular}{|c|c|c|c|c|}
\hline \multirow{2}{*}{ Variable } & \multicolumn{3}{|c|}{ Couples de même sexe } & \multirow{2}{*}{$\begin{array}{l}\text { Couples de sexe } \\
\text { différent }\end{array}$} \\
\hline & Ensemble & Couple gay & Couple lesbien & \\
\hline \multicolumn{5}{|l|}{ Statut du couple } \\
\hline Mariage/Partenariat & 15,9 & 19,9 & 11,8 & 53,6 \\
\hline Non contractualisé & 77,1 & 74,3 & 80,0 & 43,3 \\
\hline Inconnu & 7,0 & 5,8 & 8,2 & 3,1 \\
\hline \multicolumn{5}{|l|}{ Enfant(s) commun(s) } \\
\hline Oui & 6,5 & 3,6 & 9,5 & 56,7 \\
\hline Non & 93,5 & 96,4 & 90,5 & 43,3 \\
\hline \multicolumn{5}{|c|}{ Enfant(s) d'une précédente union } \\
\hline Oui & 17,2 & 12,5 & 22,0 & 20,5 \\
\hline Non & 82,8 & 87,5 & 78,0 & 79,5 \\
\hline \multicolumn{5}{|c|}{ Écart d'âge des partenaires } \\
\hline $0-4$ ans & 55,2 & 53,8 & 56,6 & 67,4 \\
\hline $5-8$ ans & 21,7 & 20,7 & 22,7 & 19,2 \\
\hline 9 ans et plus & 18,9 & 21,2 & 16,7 & 10,6 \\
\hline Inconnu & 4,2 & 4,4 & 4,0 & 2,8 \\
\hline \multicolumn{5}{|l|}{ Précédent partenaire } \\
\hline Oui & 39,9 & 32,3 & 47,7 & 27,6 \\
\hline De sexe différent & 9,6 & 10,0 & 9,3 & 27,6 \\
\hline De même sexe & 30,3 & 22,3 & 38,4 & 0,0 \\
\hline Non & 60,1 & 67,7 & 52,3 & 72,4 \\
\hline \multicolumn{5}{|l|}{ Génération } \\
\hline Avant 1960 & 18,4 & 17,6 & 19,2 & 18,6 \\
\hline 1960-1969 & 39,9 & 46,2 & 33,5 & 39,0 \\
\hline 1970-1979 & 29,0 & 27,8 & 30,3 & 30,8 \\
\hline Après 1979 & 12,7 & 8,4 & 17,0 & 11,6 \\
\hline \multicolumn{5}{|l|}{ Niveau d'instruction } \\
\hline Primaire & 2,6 & 2,7 & 2,5 & 2,2 \\
\hline Secondaire inférieur & 10,9 & 9,8 & 12,0 & 15,1 \\
\hline Secondaire supérieur & 35,0 & 32,8 & 37,3 & 42,2 \\
\hline Universitaire & 49,7 & 54,3 & 45,1 & 37,5 \\
\hline Inconnu & 1,8 & 0,4 & 3,1 & 3,0 \\
\hline \multicolumn{5}{|c|}{ Séparation des parents avant l'âge de 18 ans } \\
\hline Oui & 18,1 & 10,7 & 25,6 & 13,0 \\
\hline Non & 76,8 & 84,5 & 69,1 & 81,0 \\
\hline Inconnu & 5,1 & 4,8 & 5,3 & 6,0 \\
\hline Effectif & 385 & 170 & 215 & 36090 \\
\hline \multicolumn{5}{|c|}{$\begin{array}{l}\text { Note : Pourcentage pondéré par pays. } \\
\text { Champ : Couples corésidents formés dès 1980, par des individus âgés de } 60 \text { ans et moins. } \\
\text { Source : GGS Vague } 1 .\end{array}$} \\
\hline
\end{tabular}

qui ont officialisé leur relation et des unions libres. La proportion de couples de même sexe aurait pu être légèrement supérieure dans l'enquête GGS du fait que les couples de même sexe officialisent moins leur relation et n'apparaissent donc pas dans certaines statistiques officielles, contrairement à l'enquête GGS 
où ils sont repérés (Banens, 2010). Parmi les couples de même sexe identifiés subsistent également de «faux » couples que la méthode de correction n’aura pas relevés. Enfin, des réticences à déclarer des unions de même sexe en raison du possible stigmate social peut entraîner une sous-estimation du nombre de ces couples (Régnier-Loilier, 2018a).

En tenant compte de ces limites, il est possible de mettre en lumière plusieurs éléments distinguant les deux types de couples. Les partenaires de même sexe ont moins souvent formalisé leur union que les partenaires de sexe différent, respectivement 15,9\% et 53,6\% (tableau 3). La part de statut matrimonial inconnu qui est de 7,0\% pour les couples de même sexe provient certainement du flou légal qui peut accompagner le processus de reconnaissance de l'union par lequel le couple est passé, ainsi que les changements de législation au cours des deux dernières décennies.

Sans surprise, la présence d'enfants au sein du couple est beaucoup plus courante chez les partenaires de sexe différent : 56,7\% ont des enfants en commun auxquels s'ajoutent ceux ayant seulement des enfants issus d'une précédente relation. Les couples lesbiens sont $9,5 \%$ à avoir eu des enfants au sein de leur union. Chez 20,5\% des couples de sexe différent, au moins un des partenaires a eu un enfant dans une union antérieure. C'est le cas de $17,2 \%$ pour l'ensemble des unions de même sexe, une proportion presque égale à celle des couples hétérosexuels. La proportion de couples sans enfant est par contre près de 2,5 fois supérieure chez les couples de même sexe (78,2 \% et 32,1\%). Le moyen le plus courant d'être parent pour les partenaires de même sexe est donc d'avoir connu une précédente relation hétérosexuelle ayant mené à une naissance (Moore et Stambolis-Ruhstorfer, 2013; Gross et al., 2014).

Le parcours conjugal est plus souvent marqué par des unions antérieures pour les couples de même sexe (39,9\%), parfois avec un partenaire de sexe différent (9,6\%). L'écart d'âge entre les deux partenaires est en moyenne plus élevé chez les couples homosexuels. La séparation des parents a été plus fréquente chez les femmes en couple lesbien. Dans 25,6\% des cas, la répondante a connu la séparation de ses parents durant son enfance. Cette proportion de rupture parentale est élevée par rapport à celle des hommes gays $(10,7 \%)$ ou des hétérosexuels (13,0\%). Le niveau d'instruction des répondants en couple homosexuel est en moyenne plus élevé que celui des répondants en couple hétérosexuel. Avoir un diplôme universitaire concerne un individu sur deux en couple de même sexe (en moyenne plus jeune au moment de l'enquête). Les personnes en couple de sexe différent ont plus souvent un diplôme de secondaire supérieur, pour 42,2\% d'entre eux.

\section{Modèles de durée, risques de rupture}

Une fonction de survie représente les probabilités qu'ont les unions corésidentes de se terminer au bout de plusieurs mois (figure 1). Très clairement, 
les individus engagés dans un couple homosexuel se séparent plus rapidement que les personnes en couple hétérosexuel. Au bout de 10 mois, $10 \%$ des couples de même sexe ne vivent plus ensemble. Ce pourcentage est atteint par les couples de sexe différent après 21 mois. Après 5 ans, près de $50 \%$ des couples homosexuels se sont séparés mais seulement $25 \%$ des couples hétérosexuels ${ }^{(6)}$.

Figure 1. Probabilité de survie des couples selon leur composition sexuée

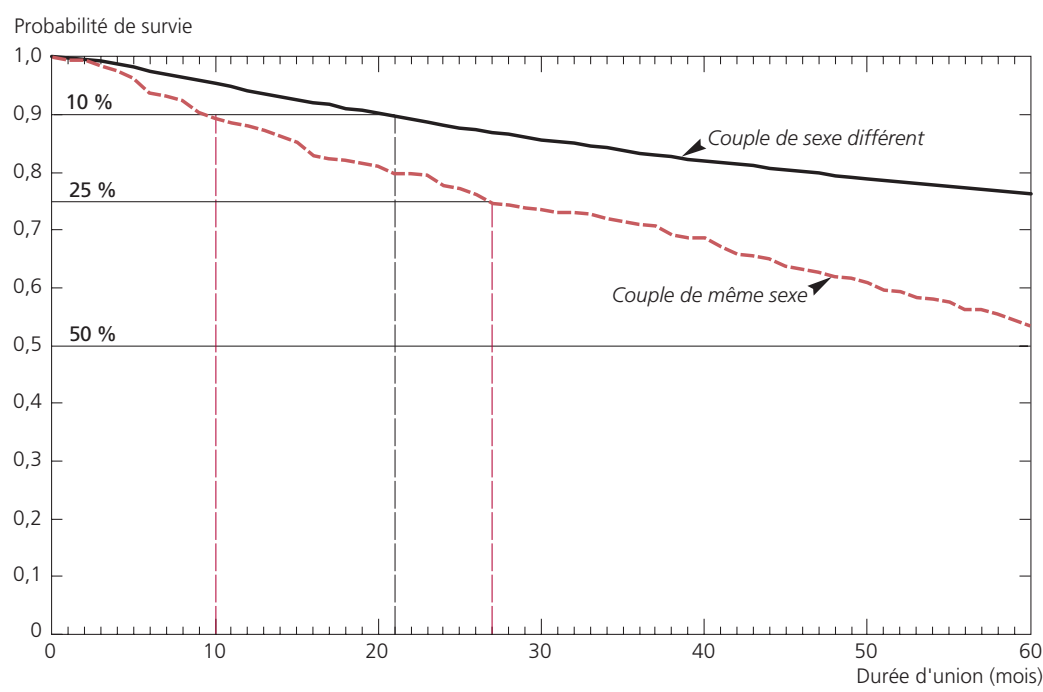

Note : Les pourcentages indiquent la proportion d'unions rompues après une durée déterminée. Lecture : $10 \%$ des couples corésidents composés de deux partenaires de sexe différent se sont séparés au bout de 21 mois.

Champ : Couples corésidents formés dès 1980, par des individus âgés de 60 ans et moins. Source: GGS Vague 1.

Les quotients de séparation (figure 2) des couples hétérosexuels augmentent légèrement lors des 12 premiers mois puis diminuent sur le long terme, autrement dit, plus les partenaires restent ensemble longtemps, moins ils ont de probabilités de se séparer. En revanche, les risques de rupture des couples de même sexe ne diminuent pas avec la durée de l'union. Après 3 ans d'union (36 mois), les quotients ont même tendance à repartir à la hausse. Le quotient de séparation pour l'ensemble des 5 premières années d'union est de 4,6 \%o [IC : 4,5-4,7\%o] pour les couples de sexe différent et 10,2 \%o [IC : 8,6-12,2\%o] pour les couples de même sexe. Ces résultats montrent donc une relative stabilité des couples hétérosexuels par rapport aux couples homosexuels, qu'il faut néanmoins nuancer par des analyses plus approfondies.

(6) Ces probabilités de survie ont également été calculées pour la première union corésidente uniquement (non présenté) et indiquent les mêmes tendances. 
Figure 2. Quotients de séparation (\%) par durée d'union et composition sexuée des couples

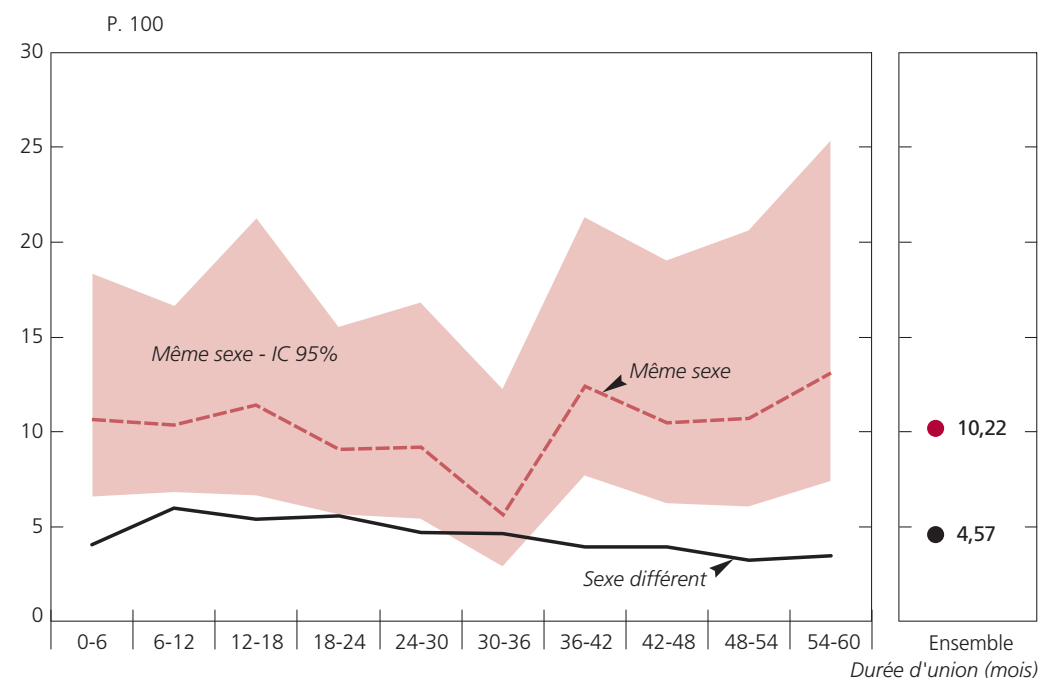

Note : Les durées d'union sont regroupées par 6 mois pour réduire la variance. Les intervalles de confiance plus réduits pour les couples de sexe différent ne sont pas présentés pour améliorer la lisibilité du graphique. Champ : Couples corésidents formés dès 1980, par des individus âgés de 60 ans et moins. Source : GGS Vague 1.

\section{Les facteurs de la séparation}

\section{L'influence du contexte légal et du statut matrimonial}

Les différences de séparation entre les couples de même sexe et de sexe différent dépendent des effets de composition de ces groupes. Le tableau 4 présente six modèles qui incluent les différents déterminants du risque de séparation. Les couples de même sexe ont un risque brut 2,37 fois plus élevé de connaître une séparation que les couples de sexe différent, confirmant la première hypothèse. Les unions composées de deux femmes sont les plus à risque de se terminer par rapport aux couples de sexe différent, avec un risque de $25 \%$ supérieur aux unions d'hommes (modèle 2). Les risques de séparation se réduisent entre unions de même sexe et de sexe différent (risque relatif (RR) : 1,64 fois), dès lors que l'on introduit dans le modèle le statut du couple (marié/en partenariat ou non) et la possibilité légale de se marier pour les couples de même sexe (modèle 3).

Un terme d'interaction entre le type de couple et le mariage/partenariat (modèle 4) permet de déterminer si l'effet de la contractualisation de l'union sur la rupture est identique entre les couples de même sexe et les couples de sexe différent. Dans ce modèle, le paramètre du type de couple représente les risques de séparation des couples de même sexe parmi l'ensemble des couples mariés. Le terme d'interaction précise si l'effet du mariage n'est pas le même entre les couples de sexe différent et de même sexe. Pour les couples de sexe 
Tableau 4. Risque relatif de séparation d'unions corésidentes (modèle de Cox)

\begin{tabular}{|c|c|c|c|c|c|c|}
\hline & Modèle 1 & Modèle 2 & Modèle 3 & Modèle 4 & Modèle 5 & Modèle 6 \\
\hline \multicolumn{7}{|c|}{ Type de couple (Réf. $=$ Sexe différent) } \\
\hline De même sexe & $2,37 * * *$ & & $1,64 * * *$ & $1,60 * * *$ & $1,49 * * *$ & \\
\hline Couple d'hommes & & $2,12 * * *$ & & & & $1,30 *$ \\
\hline Couple de femmes & & $2,65 * * *$ & & & & $1,72 * * *$ \\
\hline \multicolumn{7}{|c|}{ Statut du couple dep (Réf. $=$ Non contractualisé) } \\
\hline Mariage/partenariat & & & $0,26 * * *$ & $0,26 * * *$ & $0,34 * * *$ & $0,34 * * *$ \\
\hline $\begin{array}{l}\text { De même sexe } X \\
\text { Mariage/partenariat dep }\end{array}$ & & & & $1,74^{*}$ & & \\
\hline \multicolumn{7}{|c|}{ Mariage/partenariat homosexuel dep (Réf.= Non légal) } \\
\hline Légal & & & $1,24 * * *$ & $1,24 * * *$ & 0,99 & 0,99 \\
\hline \multicolumn{7}{|c|}{ Enfant(s) commun(s) ${ }^{\text {dep }}$ (Réf. $=$ Non) } \\
\hline Oui & & & & & $0,46 * * *$ & $0,46 * * *$ \\
\hline \multicolumn{7}{|c|}{ Enfant(s) d'une précédente relation (Réf. $=$ Non) } \\
\hline Oui & & & & & $1,12 * * *$ & $1,12 * * *$ \\
\hline \multicolumn{7}{|c|}{ Différence d'âge (Réf. = 0-4 ans) } \\
\hline $5-8$ ans & & & & & 1,00 & 1,00 \\
\hline 9 ans et plus & & & & & $1,23 * * *$ & $1,23 * * *$ \\
\hline Inconnu & & & & & $2,97 * * *$ & $2,97 * * *$ \\
\hline \multicolumn{7}{|c|}{ Âge d'entrée en union (Réf. = plus de 35 ans) } \\
\hline Moins de 25 ans & & & & & $1,38 * * *$ & $1,38 * * *$ \\
\hline $25-35$ ans & & & & & 1,06 & 1,06 \\
\hline \multicolumn{7}{|c|}{ Séparation des parents avant l'âge de 18 ans (Réf. $=$ Non) } \\
\hline Oui & & & & & $1,27 * * *$ & $1,27 * * *$ \\
\hline Inconnu & & & & & $1,60 * * *$ & $1,60 * * *$ \\
\hline \multicolumn{7}{|c|}{ Précédente relation (Réf. $=$ Non) } \\
\hline Oui & & & & & 1,05 & 1,05 \\
\hline \multicolumn{7}{|c|}{ Génération (Réf. = Avant 1960) } \\
\hline $1960-1969$ & & & & & $1,31 * * *$ & $1,31 * * *$ \\
\hline 1970-1979 & & & & & $1,66 * * *$ & $1,66 * * *$ \\
\hline Après 1979 & & & & & $2,94 * * *$ & $2,94 * * *$ \\
\hline \multicolumn{7}{|c|}{ Niveau d'éducation (Réf. = Secondaire supérieur) } \\
\hline Primaire & & & & & 1,15 & 1,15 \\
\hline Secondaire inférieur & & & & & $1,08^{*}$ & $1,08^{*}$ \\
\hline Universitaire & & & & & 1,01 & 1,01 \\
\hline Inconnu & & & & & 1,08 & 1,08 \\
\hline $\mathrm{N}$ couples & & & 364 & & & \\
\hline N séparations & & & 132 & & & \\
\hline Wald Chi $^{2}$ & 782,50 & 809,37 & 4076,51 & 4068,24 & 6718,11 & 6749,32 \\
\hline \multicolumn{7}{|c|}{$\begin{array}{l}\text { (dep) Variable dépendante du temps. } \\
\text { Notes:Tous les modèles sont contrôlés par le pays d'enquête. Le statut du couple dont la valeur est inconnue } \\
\text { est considéré comme non marié dans les modèles. } \\
\text { Significativité statistique: }{ }^{* *} p<0,001 ;{ }^{* *} p<0,01 ;{ }^{*} p<0,05 \text {. } \\
\text { Champ: Couples corésidents formés dès } 1980, \text { par des individus âgés de } 60 \text { ans et moins. } \\
\text { Source: GGS Vague } 1 \text {. }\end{array}$} \\
\hline
\end{tabular}


différent, le mariage réduit grandement les risques de séparation ( $R R$ : 0,26). L'importance de l'effet du mariage sur la séparation est néanmoins différente entre les couples de même sexe et les couples de sexe différent. La contractualisation de l'union protège de la séparation pour les deux types de couple, mais de manière moins forte pour les couples de même sexe ( $R R$ : 0,26 x 1,74=0,45). Cela semble appuyer l'hypothèse d'un impact différencié de la formalisation de l'union sur les risques de séparation pour les couples homosexuels, même si les couples qui se marient durent globalement plus longtemps que les autres. Le nombre de couples de même sexe mariés ou en union civile est cependant trop faible (60) dans l'enquête pour en tirer des conclusions solides. Le terme d'interaction sera ensuite mis de côté dans les autres modèles avec variables de contrôle.

Le mariage n'a pas été accessible pendant longtemps pour les couples de même sexe, et les risques de rupture ne peuvent être strictement comparés pour des couples s'étant formés à des dates antérieures à la légalisation du mariage homosexuel. Des analyses complémentaires (tableau annexe A.1) se limitant aux couples formés après l'introduction du mariage homosexuel (Belgique, Norvège, Pays-Bas, Suède) indiquent des résultats similaires aux modèles présentés. Cela confirme l'effet stabilisateur moins important du mariage pour les couples de même sexe que pour ceux de sexe différent.

Dans les modèles 5 et 6 , toutes les autres variables explicatives sont incluses. Le risque de séparation est toujours supérieur chez les couples de même sexe, mais de manière moins marquée (RR : 1,49 fois). Les unions composées de deux femmes sont les moins stables par rapport aux couples de sexe différent (RR : 1,72 fois par rapport aux couples de sexe différent).

\section{L'influence de la présence d'enfants}

Parmi les variables explicatives ajoutées dans les modèles 5 et 6 , la présence d'enfants communs au couple diminue les risques de rupture pour l'ensemble des unions. Par contre, avoir des enfants d'unions antérieures augmente légèrement le risque de séparation. Cela met en évidence le fait qu'un enfant issu d'une précédente relation n'a pas le même effet sur les dynamiques conjugales qu'un enfant commun.

Pour évaluer plus en détail les effets de la parentalité selon le type d'union, le recours à de nouveaux termes d'interaction permet de mesurer l'impact spécifique de la présence d'enfants communs au couple ou issus d'une précédente relation sur les risques de rupture des couples de même sexe et de sexe différent (tableau 5).

Les modèles 1 et 2 , qui ne prennent en compte que l'effet brut de la parentalité sur les risques de rupture (sans variable de contrôle), indiquent un risque nettement moindre de rupture en présence d'enfants communs au couple, et pas d'effet significatif de la présence d'enfants issus d'une précédente relation. Le modèle 3 montre que, parmi l'ensemble des couples de sexe différent, la présence 
d'enfants communs diminue sensiblement (deux fois moins) les risques de séparation (RR : 0,46). En revanche, pour les couples de même sexe, la présence - rare- d'enfants communs a un effet déstabilisateur sur l'union (RR : 0,46 x 3,55 = 1,63). La présence d'enfants issus d'une précédente union (modèle 4) augmente le risque de séparation chez les couples de sexe opposé (RR : 1,13) à caractéristiques données. Le terme d'interaction montre néanmoins que c'est à nouveau l'inverse chez les couples de même sexe. Avoir un ou des enfants issus d'une précédente relation diminue presque de moitié le risque de séparation parmi les couples de même sexe (RR : 1,13 x 0,51 =0,58). Ces résultats révèlent que les enfants issus d'un projet parental commun au couple vont avoir une influence spécifique sur la longévité de l'union en fonction de la composition sexuée du couple, tout comme les enfants issus d'une relation antérieure.

Tableau 5. Risque relatif de séparation d'unions corésidentes, interactions avec la présence d'enfants communs ou issus d'une précédente relation (modèle de Cox)

\begin{tabular}{|c|c|c|c|c|}
\hline & Modèle 1 & Modèle 2 & Modèle 3 & Modèle 4 \\
\hline \multicolumn{5}{|c|}{ Type de couple (Réf. = Sexe différent) } \\
\hline De même sexe & $1,75^{* * *}$ & $2,37 * * *$ & $1,38 * * *$ & $1,66 * * *$ \\
\hline \multicolumn{5}{|c|}{ Enfant(s) commun(s) ${ }^{\text {dep }}$ (Réf. $=$ Non) } \\
\hline Oui & $0,34 * * *$ & & $0,46 * * *$ & $0,46 * * *$ \\
\hline \multicolumn{5}{|c|}{ Enfant(s) d’une précédente relation (Réf. = Non) } \\
\hline Oui & & 1,04 & $1,12 * * *$ & $1,13 * \star \star$ \\
\hline De même sexe $X$ enfant commun & & & $3,55^{* * *}$ & - \\
\hline $\begin{array}{l}\text { De même sexe } X \text { enfant } \\
\text { d'une précédente relation }\end{array}$ & & & - & $0,51 *$ \\
\hline \multicolumn{5}{|c|}{ Statut du couple dep (Réf. $=$ Non contractualisé) } \\
\hline Mariage/partenariat & & & $0,34 * * *$ & $0,34 * * *$ \\
\hline \multicolumn{5}{|c|}{ Mariage/partenariat homosexuel dep (Réf. $=$ Non légal) } \\
\hline Légal & & & 0,99 & 0,99 \\
\hline Variables de contrôle & Non & Non & Oui & Oui \\
\hline $\mathrm{N}$ couples & \multicolumn{4}{|c|}{36475} \\
\hline $\mathrm{N}$ séparations & \multicolumn{4}{|c|}{13287} \\
\hline Wald $\mathrm{Chi}^{2}$ & 2673,47 & 790,58 & 6734,85 & 6802,12 \\
\hline \multicolumn{5}{|c|}{$\begin{array}{l}\text { (dep) : Variable dépendante du temps. } \\
\text { Notes:Tous les modèles sont contrôlés par le pays d'enquête. Variables de contrôle : différence d'âge, séparation } \\
\text { des parents, âge d'entrée en union, génération, niveau d'éducation, précédente relation. Le statut du couple } \\
\text { dont la valeur est inconnue est considéré comme non marié dans les modèles. } \\
\text { Significativité statistique : }{ }^{* *} p<0,001 ;{ }^{* *} p<0,01 \text { * }^{*} p<0,05 \text {. } \\
\text { Champ : Couples corésidents formés dès } 1980, \text { par des individus âgés de } 60 \text { ans et moins. } \\
\text { Source: GGS Vague } 1 \text {. }\end{array}$} \\
\hline
\end{tabular}

Notre troisième hypothèse est ainsi réfutée : la parentalité ne suffit pas à expliquer les différences résiduelles de séparation entre les deux types de couples. Elle produit même des effets inverses sur la rupture entre couples de même sexe et de sexe différent, en fonction de la présence d'enfants communs ou issus d'une précédente relation. 
Bien que ces modèles proposent une manière d'isoler l'effet de la parentalité sur le risque de séparation, ils présentent une limite majeure : pour les couples hétérosexuels, l'absence d'enfant commun peut être révélatrice d'une divergence de préférences quant à la parentalité, ou une infertilité de l'un des deux membres du couple, qui amènent des risques accrus de séparation à la suite d'un projet parental inabouti. Cette question du désir d'enfant se pose moins chez les couples de même sexe, dont l'accès difficile à la parentalité peut décourager la plupart d'entre eux d'avoir un enfant commun. Cela se traduit dans notre échantillon par un nombre limité (25) de couples de même sexe avec enfant commun, invitant à nouveau à la prudence dans les conclusions associées à cet effet.

\section{Conclusion}

Dans ce travail, l'enquête Generations and Gender Survey combinée pour six pays européens a été utilisée afin de mesurer la stabilité des couples corésidents, selon leur composition sexuée. Il s'agissait d'estimer si les couples corésidents de même sexe avaient des risques de rupture plus importants que les couples corésidents de sexe différent, et d'en analyser les déterminants. Le mariage et la présence d'enfants, longtemps inaccessibles pour les couples de même sexe, ont ensuite été étudiés comme sources de différences entre les deux types de couples par rapport aux risques de rupture.

Nos résultats montrent que les unions de même sexe se terminent plus souvent que les unions de sexe différent. Cinq ans après le début de leur union, près de $50 \%$ des couples homosexuels se sont séparés, contre $25 \%$ des couples hétérosexuels. Des effets de composition de groupe et de sélection sont cependant importants. Ainsi, être marié et avoir des enfants avec son conjoint - caractéristiques plus fréquentes chez les couples de sexe différent-réduisent les risques de séparation. Toutefois, ces deux caractéristiques, lorsqu'elles sont présentes chez les couples de même sexe, n'affectent pas les risques de séparation de la même manière.

Pour l'ensemble des couples, l'absence de cadre légal dans l'union permet de la rompre plus rapidement. Néanmoins, la formalisation de l'union a une influence moins importante sur la stabilité des couples de même sexe que chez ceux de sexe différent, le mariage (ou partenariat) n'explique pas totalement les différences de risques de rupture entre les deux types de couples. Les normes conjugales entre personnes de même sexe ne sont pas définies aussi clairement et depuis aussi longtemps que pour les couples de sexe différent, ce qui laisse imaginer que le mariage ou le partenariat enregistré peut être investi en ayant une moindre influence sur la stabilité de l'union (Lerch, 2007). Dès lors, les différences de stabilité conjugale entre couples de même sexe mariés et non mariés seraient réduites. 
Certains couples de même sexe, même s'ils en ont la possibilité, ne voient pas le mariage comme un facteur d'engagement primordial, d'autres moyens étant mis en place pour formaliser leur union, à travers une cérémonie par exemple (Reczek et al., 2009). D'autres vont rejeter les normes associées au mariage et privilégier la non-contractualisation de leur union, ou alors la contractualisation par le partenariat enregistré, s'il se distingue du mariage (Jörgens, 2008; Courduriès, 2011; Rault, 2018). L'emménagement dans un domicile commun peut lui-même constituer un engagement fort dans la conjugalité, au vu de la proportion plus importante de couples de même sexe (surtout gays) qui ne vivent pas sous le même toit (Courduriès, 2011; Rault, 2018). La signification du mariage, de l'union civile et de la cohabitation est variable pour les couples de même sexe. La légalisation récente du mariage homosexuel ajoute toutefois un élément nouveau dans la trajectoire conjugale des partenaires de même sexe. Dans les pays qui l'autorisent, les couples de même sexe doivent désormais se positionner par rapport à la possibilité de formaliser leur union (Courduriès, 2011). De futures études permettront, avec un recul plus important sur cette légalisation, de déterminer si le mariage et l'union civile sont de plus en plus adoptés par les couples de même sexe, si les normes évoluent et si cela aura un impact différent sur le risque de rupture dans l'avenir.

La parentalité chez les couples de même sexe est un autre élément dont les normes ne se dessinent que depuis peu. Elle a une influence spécifique sur la rupture. Alors que la présence d'enfants communs chez les couples de sexe différent est synonyme d'une longévité accrue de l'union, c'est l'effet inverse pour les couples de même sexe. En revanche, les enfants issus d'une précédente relation chez les couples de même sexe semblent avoir une influence positive sur la longévité de l'union. Les couples de même sexe, pour être parents, doivent passer par différentes dispositions légales en fonction du pays, comme l'adoption, la gestation pour autrui ou la procréation médicalement assistée pour les couples de femmes (Gross et al., 2014). Ce processus est long, certaines tentatives pouvant s'avérer infructueuses, et rend l'accès à la parentalité moins immédiat que pour les couples de sexe différent. Si un enfant naît ou est adopté, la conciliation entre vie familiale et vie professionnelle pourrait également être plus difficile pour les couples de même sexe. En l'absence de rôles de genre prédéfinis et dans une optique égalitaire, les couples de même sexe auraient plus de contraintes d'organisation de la vie familiale et parentale, pouvant favoriser l'instabilité conjugale (Wiik et al., 2014). Un enfant issu d'une précédente union ne pose pas autant cette question de l'entrée en parentalité et de division du travail, puisqu'il provient d'un projet parental qui ne concerne pas le couple actuel. Pour au moins l'un des deux partenaires, cette forme de parentalité s'intègre dans une trajectoire conjugale où une union hétérosexuelle féconde précède la formation d'un couple de même sexe. Les valeurs familiales traditionnelles acquises avec le partenaire de sexe opposé pourraient se perpétuer avec le partenaire de même sexe, apportant une stabilité dans cette nouvelle union. 
Même en tenant compte des effets de composition des deux types de couples, et particulièrement des effets du mariage et de la présence d'enfants, le risque de séparation reste supérieur pour les couples de même sexe. En distinguant couples de femmes et couples d'hommes, les couples lesbiens sont les plus à risque de se séparer par rapport aux couples hétérosexuels. Cela pourrait tenir au fait que les femmes sont davantage sensibles à la qualité de la relation que les hommes, en étant par exemple plus souvent à l'origine d'un divorce dans les couples de sexe différent (Kalmijn et Poortman, 2006; Kurdek, 2006). La durée entre la rencontre de la partenaire et la mise en union corésidente peut être une autre interprétation, car elle est plus courte pour les couples lesbiens que pour les couples gays, facteur révélateur d'un engagement rapide dans une relation de couple cohabitant, mais qui pourrait favoriser l'instabilité (Lau, 2012; Rault, 2018).

D'autres facteurs non observables directement dans l'enquête GGS pourraient être à l'origine des différences résiduelles de longévité des unions selon la composition sexuée du couple. Parmi ces facteurs non observables, l'hétéronormativité et la stigmatisation des couples homosexuels qui en découle peuvent avoir un impact sur la stabilité de l'union de deux partenaires de même sexe. Bien que l'acceptation sociale de la conjugalité homosexuelle soit un phénomène qui s'amplifie avec les années, se confronter à la norme hétérosexuelle peut être une expérience ardue, surtout si elle est associée à un manque de soutien social (Jörgens, 2008; Van Eeden-Moorefield et al., 2011). Le fait de se marier pourrait cependant être une stratégie adoptée par ces couples pour acquérir plus de reconnaissance sociale de la part de l'entourage.

Cette recherche présente certaines limites, dont les principales sont les erreurs résiduelles d'encodage du sexe dans l'enquête, le nombre restreint de couples de même sexe dans nos analyses statistiques, et la période d'observation qui limite la comparaison entre le type de couple et toutes les formes de contractualisation de l'union. Le recours à des sources de données d'origine longitudinale et administrative (registre, données fiscales annuelles) offre des perspectives intéressantes en termes de repérage d'000erreurs d'encodage du sexe. Celles-ci seront réduites par rapport aux enquêtes ou aux recensements de population, car le sexe des individus est vérifié par le biais des administrations utilisant ces données. L'identification des couples de même sexe non mariés y est par contre plus compliquée, peu d'informations étant disponibles sur la nature du lien qui unit deux personnes cohabitantes non apparentées (Festy, 2007). La prise en compte des enjeux méthodologiques associés à la mesure des unions de même sexe demeure primordiale dans toutes les recherches quantitatives tentant de dégager des tendances spécifiques aux couples de même sexe. En prime, ces données permettront d'intégrer de nouvelles dimensions explicatives des trajectoires conjugales, comme les trajectoires résidentielles pour les registres nationaux ou les trajectoires 
professionnelles avec les données fiscales. Elles contribueront à étendre les analyses de facteurs de stabilité des unions, et ainsi intégrer la variété de situations conjugales et familiales qui caractérise l'Europe de ce début du $\mathrm{XXI}^{\mathrm{e}}$ siècle.

Remerciements : Je tiens à remercier Ester Rizzi d'avoir accepté de veiller au bon déroulement de ce projet de recherche. Merci à Philippe Bocquier pour ses connaissances et son appui sur les modèles d'analyse longitudinale. Ce travail n'aurait également pas eu la même valeur sans l'aide d'Arnaud Régnier-Loilier, qui m’a permis de saisir l'enjeu derrière la mesure des couples de même sexe. Lauteur remercie les rédactrices et les relecteurs pour leurs commentaires précieux.

Les données ont été obtenues à partir du « Generations and Gender Programme Data Archive ", en respect des normes de confidentialité. 

ANNEXE 
Tableau A.1. Risque relatif de séparation d'unions corésidentes, champ des unions formées après introduction du mariage homosexuel (modèle de Cox)

\begin{tabular}{|c|c|c|c|c|}
\hline & Modèle $1 b$ & Modèle 3b & Modèle $4 b$ & Modèle $5 b$ \\
\hline \multicolumn{5}{|c|}{ Type de couple (Réf. $=$ Sexe différent) } \\
\hline De même sexe & $1,74 * \star *$ & $1,54^{* *}$ & $1,45^{\star *}$ & $1,48^{* *}$ \\
\hline \multicolumn{5}{|c|}{ Statut du couple dep $($ Réf. $=$ Non marié) } \\
\hline Mariage & & $0,25 * * *$ & $0,24 * * *$ & $0,33 * * *$ \\
\hline De même sexe $X$ Mariage dep & & & $2,66^{*}$ & \\
\hline \multicolumn{5}{|c|}{ Enfant(s) commun(s) dep (Réf. = Non) } \\
\hline Oui & & & & $0,36 * * *$ \\
\hline \multicolumn{5}{|c|}{ Enfant(s) d'une précédente relation (Réf. $=$ Non) } \\
\hline Oui & & & & $1,14^{*}$ \\
\hline \multicolumn{5}{|c|}{ Différence d'âge (Réf. $=0-4$ ans) } \\
\hline $5-8$ ans & & & & 0,91 \\
\hline 9 ans et plus & & & & 1,12 \\
\hline Inconnu & & & & $1,92 * * *$ \\
\hline \multicolumn{5}{|c|}{ Âge d'entrée en union (Réf. = plus de 35 ans) } \\
\hline Moins de 25 ans & & & & $1,33 * *$ \\
\hline $25-35$ ans & & & & 0,97 \\
\hline \multicolumn{5}{|c|}{ Séparation des parents avant l'âge de 18 ans (Réf. $=$ Non) } \\
\hline Oui & & & & $1,11 *$ \\
\hline Inconnu & & & & $2,29 * * *$ \\
\hline \multicolumn{5}{|c|}{ Précédente relation (Réf. = Non) } \\
\hline Oui & & & & 0,92 \\
\hline \multicolumn{5}{|c|}{ Génération (Réf. = Avant 1960) } \\
\hline 1960-1969 & & & & $1,93 * * *$ \\
\hline 1970-1979 & & & & $2,09 * * *$ \\
\hline Après 1979 & & & & $3,01 * * *$ \\
\hline \multicolumn{5}{|c|}{ Niveau d'éducation (Réf. = Secondaire supérieur) } \\
\hline Primaire & & & & 0,97 \\
\hline Secondaire inférieur & & & & $1,42 * * *$ \\
\hline Tertiaire & & & & $1,19 * * *$ \\
\hline Inconnu & & & & 1,20 \\
\hline $\mathrm{N}$ couples & & & & \\
\hline $\mathrm{N}$ séparations & & & & \\
\hline Wald Chi ${ }^{2}$ & 177,94 & 694,49 & 688,19 & 1649,97 \\
\hline \multicolumn{5}{|c|}{$\begin{array}{l}\text { (dep) : variable dépendante du temps. } \\
\text { Notes: Tous les modèles contrôlent pour le pays d'enquête (Belgique, Norvège, Pays-Bas, Suède). Les modèles } 2 \\
\text { et } 6 \text { du tableau } 4 \text { ne sont pas pris en compte. } \\
\text { Significativité statistique: } * * * p<0,001 ;{ }^{* *} p<0,01 ;{ }^{*} p<0,05 \text {. } \\
\text { Champ: Couples corésidents formés depuis la légalisation du mariage homosexuel selon le pays d'enquête, } \\
\text { pour des individus âgés de } 60 \text { ans et moins. } \\
\text { Source: GGS Vague } 1 .\end{array}$} \\
\hline
\end{tabular}




\section{RÉFÉRENCES}

Andersson G., NOACK T., Seierstad A., WeEdOn-FEKJÆr H., 2006, The demographics of same-sex marriages in Norway and Sweden, Demography, 43(1), p. 79-98.

Andress H.-J., BORGlOH B., BröCKel M., Giesselmann M., Hummelsheim D., 2006, The economic consequences of partnership dissolution: A comparative analysis of panel studies from Belgium, Germany, Great Britain, Italy, and Sweden, European Sociological Review, 22(5), p. 533-560.

BANENS M., 2010, Mariage et partenariat de même sexe en Europe [Vingt ans d'expérience], Politiques sociales et familiales, 99, p. 73-84.

BECKER G., 1981, A Treatise on the Family, Cambridge, Harvard University Press.

BLACKWELL D., LICHTER D., 2004, Homogamy among dating, cohabiting, and married couples, The Sociological Quaterly, 45(4), p. 719-737.

Blumstein P., SchWARTz P., 1983, American Couples: Money, Work, Sex, New York.

BOZON M., RAULT W., 2012, De la sexualité au couple. L'espace des rencontres amoureuses pendant la jeunesse, Population, 67(3), p. 453-490.

BRINES J., JOYNER K., 1999, The ties that bind: Principles of cohesion in cohabitation and marriage, American Sociological Review, 64(3), p. 333-355.

Buisson G., LAPINTE A., 2013, Le couple dans tous ses états : non-cohabitation, conjoints de même sexe, Pacs, Insee première, $\mathrm{n}^{\circ} 1435$.

COURduriès J., 2011, Être en couple (gay). Conjugalité et homosexualité masculine en France, Presses universitaires de Lyon.

FESTY P., 2006, Légaliser les unions homosexuelles en Europe : innovations et paradoxes, Population et sociétés, $\mathrm{n}^{\circ} 424$.

FESTY P., 2007, Numbering same-sex couples in censuses and population registers, Demographic Research, 17(12), p. 339-368.

Fokkema T., Kveder A., Hiekel N., Emery T., Liefbroer A., 2016, Generations and Gender Programme Wave 1 data collection: An overview and assessment of sampling and fieldwork methods, weighting procedures, and cross-sectional representativeness, Demographic Research, 34(18), p. 499-524.

Gross M., COURduriÈs J., DE FEDERICO A., 2014, Le recours à l'AMP dans les familles homoparentales : état des lieux. Résultats d'une enquête menée en 2012, Socio-logos, 2870(9), p. 1-17.

JÖRGENS F., 2008, Projets de couple, projets de famille. Discours homosexuels sur le mariage et la parentalité, in Descoutures V., Digoix M., Fassin E., Rault W. (dir.), Mariages et homosexualités dans le monde, Paris, Autrement, p. 127-137.

Kalmijn M., POORTMAn A.-R., 2006, His of her divorce? The gendered nature of divorce and its determinants, European Sociological Review, 22(2), p. 201-214.

Kalmijn M., Loeve A., Manting D., 2007, Income dynamics in couples and the dissolution of marriage and cohabitation, Demography, 44(1), p. 159-179.

KURDEK L., 1998, Relationship outcomes and their predictors: Longitudinal evidence from heterosexual married, gay cohabiting, and lesbian cohabiting couples, Journal of Marriage and Family, 60(3), p. 553-568. 
KURDEK L., 2006, Differences between partners from heterosexual, gay, and lesbian cohabiting couples, Journal of Marriage and Family, 68(2), p. 509-528.

LAU C., 2012, The stability of same-sex cohabitation, different-sex cohabitation, and marriage, Journal of Marriage and Family, 74(5), p. 973-988.

LERCH A., 2007, Normes amoureuses et pratiques relationnelles dans les couples gays, Informations sociales, 144(8), p. 108-117.

LesthaEghe R., 2010, The unfolding story of the second demographic transition, Population and Development Review, 36(2), p. 211-251.

LEVINGER G., 1965, Marital cohesiveness and dissolution: An integrative review, Journal of Marriage and Family, 27(1), p. 19-28.

LYNGSTAD T., JALOVAARA M., 2010, A review of the antecedents of union dissolution, Demographic Research, 23(10), p. 257-292.

MANNING W., BROWN S., STYKeS B., 2016, Same-sex and different-sex cohabiting couple relationship stability, Demography, 53(4), p. 937-953.

MoOre M., STAMBOLIS-RUHSTORFER M., 2013, LGBT sexuality and families at the start of the twenty-first century, Annual Review of Sociology, 39(1), p. 491-507.

Oppenheimer V., 1988, A theory of marriage timing, American Journal of Sociology, 94(3), p. 563-591.

RAULT W., 2018, La distance dans les relations conjugales et familiales des gays et des lesbiennes, in Imbert C., Lelièvre E., Lessault D. (dir.), La famille à distance, Paris, Ined, Questions de populations, p. 257-275.

ReCZeK C., ElliotT S., UMberson D., 2009, Commitment without marriage: Union formation among long-term same-sex couples, Journal of Family Issues, 30(6), p. $738-756$.

RÉGNIER-LOILIER A., 2018a, Are the Generations and Gender Surveys well suited for studying same-sex couples?, European Journal of Population, 34(4), p. 567-578.

RÉGNIER-LOILIER A., 2018b, Faire couple à distance. Prévalence et caractéristiques, in Imbert C., Lelièvre E., Lessault D. (dir.), La famille à distance, Paris, Ined, Questions de populations, p. 165-193.

ROSENFELD M., 2014, Couple longevity in the era of same-sex marriage in the United States, Journal of Marriage and Family, 76(5), p. 905-918.

VAN DE KAA D., 1987, Europe's second demographic transition, Population Bulletin, 42(1), p. 1-59.

Van Eeden-Moorefield B., Martell C., Williams M., Preston M., 2011, Samesex relationships and dissolution: The connection between heteronormativity and homonormativity, Family Relations, 60(5), p. 562-571.

Verbakel E., Kalmijn M., 2014, Assortative mating among Dutch married and cohabiting same-sex and different-sex couples, Journal of Marriage and Family, 76(1), p. 1-12.

WEISSHAAR K., 2014, Earnings equality and relationship stability for same-sex and heterosexual couples, Social Forces, p. 1-31.

WiIK K., SEIERSTAD A., NOACK T., 2014, Divorce in Norwegian same-sex marriages and registered partnerships: The role of children, Journal of Marriage and Family, 76(5), p. 919-929. 


\section{Benjamin MARTEAU • LA SÉPARATION CHEZ LES COUPLES CORÉSIDENTS DE MÊME SEXE ET DE SEXE DIFFÉRENT}

Les couples de même sexe s'inscrivent dans un schéma de diversification des situations conjugales et familiales en Europe. L'enquête Générations et genre (Generations and Gender Survey), utilisée ici pour six pays européens (Allemagne, Belgique, France, Norvège, Pays-Bas, Suède), permet d'analyser l'histoire conjugale des individus et le sexe des partenaires avec qui ils ont cohabité au cours de leur vie. Après avoir tenu compte des erreurs d'encodage du sexe inhérentes à ce type d'enquêtes, nous comparons les risques de rupture des couples formés d'un homme et d'une femme aux couples de personnes de même sexe, avec un modèle de Cox. Ces résultats montrent que les couples de même sexe présentent un risque brut plus élevé de connaître une séparation (2,4 ois) que les couples de sexe différent, mais qui se réduit lorsque les variables explicatives et de contrôle sont introduites dans les modèles. Le mariage et la présence d'enfants constituent deux éléments majeurs augmentant la longévité des couples de sexe différent, situations que les couples de même sexe connaissent moins fréquemment.

\section{Benjamin Marteau • Separation Among Cohabiting SAME-SeX AND Different-SeX COUPLES}

Same-sex couples are one component of the growing diversity of family and partnership situations in Europe. Data from six countries that took part in the Generations and Gender Survey (Belgium, France, Germany, the Netherlands, Norway, and Sweden) were used to analyse the respondents' partnership histories and the sex of current and previous cohabiting partners. After correcting for the coding errors inherent in this type of survey, a Cox model was used to compare the separation risks of different-sex and same-sex couples. The results show that the unadjusted separation risk for same-sex couples is 2.4 times higher than that for differentsex couples, but that this risk decreases after the independent and control variables are included in the model. Marriage and the presence of children, less frequent among same-sex couples, are two key factors that increase the stability of different-sex unions.

\section{Benjamin Marteau • LA SEPARACIÓN DE LAS PAREJAS CO-RESIDENTE DEL MISMO SEXO Y DE SEXO DIFERENTE,}

Las parejas homosexuales se inscriben en un esquema de diversificación de las situaciones conyugales y familiares en Europa. La encuesta Generations and Gender Survey, utilizada aquí para seis países europeos (Alemania, Bélgica, Francia, Noruega, Países-Bajos, Irlanda), permite analizar la historia conyugal de los individuos así como el sexo de los conyugues con quienes han cohabitado en el curso sus vidas. Una vez considerados los errores de codificación del sexo inherentes a este tipo de encuestas, comparamos, con un modelo de Cox, el riesgo de ruptura de las parejas formadas por un hombre y una mujer con el de las parejas del mismo sexo. Los resultados muestran que las parejas del mismo sexo presentan un riesgo bruto más elevado de separación (2,4 veces más) que las parejas de sexo diferente, pero esta diferencia se reduce cuando las variables explicativas y de control se introducen en los modelos. El matrimonio y la presencia de hijos constituyen dos elementos importantes que aumentan la longevidad de las parejas de distinto sexo, situaciones que las parejas del mismo sexo conocen con menos frecuencia.

Mots-clés : couple, couple de même sexe, cohabitation, séparation, Generations and Gender Survey

Keywords: union, same-sex couples, cohabitation, separation, Generations and Gender Survey 
